\title{
TRANSVERSE SIZE EFFECTS IN THE FRAGMENTATION OF HADRONIC STRINGS
}

\author{
K. SAILER ${ }^{\text {a.b }}$, Th. SCHÖNFELD ${ }^{a}$, A. SCHÄFER ${ }^{c}$, B. MÜLLER ${ }^{\text {a.d }}$ and W. GREINER ${ }^{a}$ \\ - Institut für Theoretische Physik, Goethe Universitäl, D-6000 Frankfurt am Main. FRG \\ - Institute for Theoretical Physics, Kossuth Liniversity, H-4010 Debrecen, Hungary \\ c Max-Planck-Institut für Kernphysik, D-6900 Heidelberg. $F R G$ \\ d Department of Physics, Duke University, Durham, NC 27706, USA \\ Received 7 February 1990
}

\begin{abstract}
The transverse momentum distribution of (anti) quarks created in the chromo-electric field of an infinitely long hadronic flux tube is derived. The effects of the finite radius of the hadronic strings on their fragmentation are studied simulating $\mathrm{e}^{+} \mathrm{e}^{-}$annihilation events in the framework of a dynamical string model that contains two parameters: the string tension $\kappa=0.89 \mathrm{GeV} / \mathrm{fm}$ (determined by the Regge slope), and the radius of the string. It is shown that a string radius of about 0.5 fm allows to describe successfully both the width of the transverse momentum distribution in the low- $p_{\mathrm{T}}$ region and the multiplicities.
\end{abstract}

\section{Introduction}

String models have been quite successful in describing quark fragmentation into hadrons, e.g. in electron-positron annihilation [1-3]. In the Lund fragmentation scheme, the string stretched between the leading quark and antiquark fragments into smaller pieces according to phenomenological scaling functions. Creation of a qã pair in the chromoelectric field of the hadronic flux tube followed by a full screening of the field between them is thought to be the underlying physical picture of the decay of hadronic strings. Although the scaling functions governing the decay have been introduced independently of the flux tube model, it has motivated the introduction of some additional effects connected with the transverse momenta of the created qa pairs: (i) The quark-antiquark pair created with compensating non-vanishing transverse momenta, has to be produced on-shell with a certain qq separation [4]. The energy of the chromoelectric field between them must be sufficient to provide the transverse energy of the created q $\bar{q}$ pair. Hence, some time must elapse until the pair created virtually at a point comes sufficiently far apart. This effect has been taken into account by introducing a weighting factor at each pair production vertex [2]. (ii) The transverse momen- tum distribution of the fragments has been chosen according to that of the qqu pairs created in an infinitely extended homogeneous field [2]. Such a transverse momentum distribution of the quarks corresponds to the $q \bar{q}$ pair production rate calculated according to Schwinger's formula [5]. Replacing the product of the electric charge with the field strength, $e \&$ by the string tension $\kappa$ one can apply this formula to the estimation of the decay constant of an excited hadron in the framework of the flux tube model [6]. The transverse momentum distribution of the created (anti)quarks is then obtained from the underlying tunneling probability of a qq̄ pair with given transverse momentum.

It is, however, clear that the chromoelectric field of the excited hadron does not extend to the whole space [7]. It is rather confined into a region of finite transverse size of the order of $1 \mathrm{fm}$. Therefore, in the present paper, we will take the decay properties of hadronic strings as they are predicted by the flux tube model when the finite radius of the tube is accounted for, but the tube is infinitely long. Recently we have derived the pair creation probability per unit time and volume in a constant electric field restricted to an infinitely long cylinder [8]. Now we derive the transverse momentum distribution of the (anti)quarks created in such a cylinder making use of the asymp- 
totic behaviour of the wave function obtained in ref. [8]. Then we apply the results to excited hadrons. They are considered as $(3+1)$-dimensional strings with respect to their motion in space, but having a non-vanishing transverse radius. We will discuss the effect of the finite radius on the observables by simulating two-jet like $\mathrm{e}^{+} \mathrm{e}^{-}$annihilation events at low CM energy $(\sqrt{s}=20 \mathrm{GcV})$ where soft fragmentation is dominating.

\section{Transverse momentum distribution}

We start with the derivation of the transverse momentum distribution of (anti)quarks with zero rest mass created in a constant chromoelectric field $\&$ restricted to an infinitely long cylinder of radius $R$. In ref. [8] it was found that the pair production probability per unit time and unit volume is given as follows:

$\mathscr{H}=\mathscr{W}_{\mathrm{s}} \mathscr{R}(R)$,

where

$$
\mathscr{H}_{\mathrm{s}}=(24 \pi)^{-1}(\mathrm{e} \mathscr{E})^{2}
$$

is the pair production rate in an infinitely extended homogeneous electric field.

$\not h(R)=-\frac{24}{(\pi u)^{2}} \sum_{n=1}^{\infty} \sum_{\mu=0}^{\infty} \ln \left[1-\exp \left(-\frac{x_{n \mu}^{2}}{u^{2}}\right)\right]$

is the correction factor due to the finite radius with $u=\sqrt{e \delta R^{2} / \pi} ; n$ plays the role of a principal quantum number and $\mu$ is the magnetic quantum number. The $x_{n \mu}$ are the roots of the linear bag boundary condition given in ref. [7]:

$J_{\mu}^{2}(x)=J_{\mu+1}^{2}(x)$.

For very large times $t \rightarrow \pm \infty$, the solution of the Dirac equation has the asymptotic form obtained in ref. [8]:

$$
\begin{aligned}
& \psi_{n \mu}(t, \rho, \phi) \sim q_{n \mu}( \pm \infty ; \rho, \phi) \exp \left( \pm \mathrm{i} \tau^{2} / 2\right) \\
& \quad+\bar{q}_{n \mu}( \pm \infty ; \rho, \phi) \exp \left(\mp \mathrm{i} \tau^{2} / 2\right),
\end{aligned}
$$

with $\tau=\sqrt{e \&}\left(1+p_{3} / e \delta\right) \sim \sqrt{e \mathscr{E}} t$ where $p_{3}$ is the longitudinal momentum of the quark. We suppressed the suffix $p_{3}$ since the dependence on the longitudinal coordinate $x_{3}$ becomes trivial in the infinite time limit. Furthermore, the functions $q_{n \mu}$ and $\bar{q}_{n \mu}$ contain a time dependent phase factor which we did not note explicitly. As it was shown in ref. [8], the function $q_{n \mu}(-\infty ; \rho, \phi)$ vanishes identically. According to eq. (5), the functions $q_{n \mu}\left(\bar{q}_{n \mu}\right)$ can be interpreted as the wave functions of the quark (antiquark) in the state $(n \mu)$. Thus the antiquark state at $t \rightarrow-\infty$ evolves to a quark-antiquark state at $t \rightarrow+\infty$. (The choice of the initial state is arbitrary. Only the change of particle numbers is relevant. Our choice is motivated by the relative simplicity of the resulting expressions.) Then the transverse momentum distributions $P_{n \mu}\left(p_{\mathrm{T}}\right)$ and $\bar{P}_{n \mu}\left(p_{\mathrm{T}}\right)$ of the created quark and antiquark, respectively, can be obtained as follows:

$P_{n \mu}\left(p_{\mathrm{T}}\right)=\left|\tilde{q}_{n \mu}\left(+\infty ; p_{\mathrm{T}}\right)\right|^{2}$,

and

$\bar{P}_{n \mu}\left(p_{\mathrm{T}}\right)=\left|\tilde{\bar{q}}_{n \mu}\left(-\infty ; p_{\mathrm{T}}\right)\right|^{2}-\left|\tilde{\bar{q}}_{n \mu}\left(+\infty ; p_{\mathrm{T}}\right)\right|^{2}$,

in terms of the Fourier transforms of the quark and antiquark wave functions, $\tilde{q}_{n \mu}\left(+\infty ; p_{\mathrm{T}}\right)$ and $\tilde{\bar{q}}_{n \mu}\left( \pm \infty ; p_{\mathrm{r}}\right)$, respectively. Introducing the integrals

$$
\begin{aligned}
& I_{n \mu, \mu^{\prime}}\left(p_{\mathrm{T}}\right)=\int_{0}^{R} \mathrm{~d} \rho \rho \\
& \times \int_{0}^{2 \pi} \mathrm{d} \phi \exp \left(\mathrm{i} \mu^{\prime} \phi\right) J_{\mu}\left(k_{n \mu} \rho\right) \exp \left(-\mathrm{i} p_{\mathrm{T}} \rho \cos \phi\right),
\end{aligned}
$$

with $k_{n \mu}=x_{n \mu} / R$, the Fourier transform of the quark wave function can be written in the following form:

$\tilde{q}_{n \mu}\left(+\infty ; p_{\mathrm{T}}\right)=v_{n \mu} k_{n \mu}\left(\begin{array}{c}f_{n \mu} I_{n \mu, \mu}\left(p_{\mathrm{T}}\right) \\ -\mathrm{i} g_{n \mu} I_{n \mu, \mu+1}\left(p_{\mathrm{T}}\right) \\ f_{n \mu} I_{n \mu, \mu}\left(p_{\mathrm{T}}\right) \\ -\mathrm{i} g_{n \mu} I_{n \mu, \mu+1}\left(p_{\mathrm{T}}\right)\end{array}\right)$,

where

$f_{n \mu}=g_{n \mu} J_{\mu+1}\left(x_{n \mu}\right) / J_{\mu}\left(x_{n \mu}\right)$,

$g_{n \mu}=h_{n \mu} \exp \left(-\frac{3}{8} \pi \hat{\lambda}_{0}\right)$,

with $\lambda_{0}=k_{n \mu}^{2} / e \delta$ and the $\tau$ dependent phase factor $h_{n \mu}$, i.e. $\left|h_{n \mu}\right|^{2}=1$. The Fourier transforms of the antiquark wave functions have a very similar form. The coefficients $f_{n \mu}$ and $g_{n \mu}$ and the corresponding ones for the antiquark wave functions were obtained by 
using the asymptotic form of the parabolic cylinder functions [9].

Inserting expression (9) into eq. (6) and making use of the boundary condition (4), we obtain

$$
P_{n \mu}\left(p_{\mathrm{T}}\right)=2 . V_{n \mu}^{2} k_{n \mu}^{2}\left[\left|I_{n \mu, \mu}\left(p_{\mathrm{T}}\right)\right|^{2}+\left|I_{n \mu, \mu+1}\left(p_{\mathrm{T}}\right)\right|^{2}\right] .
$$

The integrals ( 8 ) can be performed making use of the following integral formula [10];

$$
\begin{aligned}
& \int \mathrm{d} z z J_{\mu}(\alpha z) J_{\mu}(\beta z) \\
& \quad=\frac{\beta z J_{\mu}(\alpha z) J_{\mu-1}(\beta z)-\alpha z J_{\mu-1}(\alpha z) J_{\mu}(\beta z)}{\alpha^{2}-\beta^{2}},
\end{aligned}
$$

and of the integral representation of the Bessel functions. So we get

$$
I_{n \mu, \mu^{\prime}}=2 \pi R^{2} \mathrm{i}^{-\mu^{\prime}} \cdot \mathscr{I}_{\mu^{\prime}}\left(x_{n \mu}, p_{\mathrm{T}} R\right),
$$

with

$$
\begin{gathered}
\mathscr{f}_{\mu}(\alpha, \beta)=\frac{\beta J_{\mu}(\alpha) J_{\mu-1}(\beta)-\alpha J_{\mu-1}(\alpha) J_{\mu}(\beta)}{\alpha^{2}-\beta^{2}} \\
=\frac{\alpha J_{\mu+1}(\alpha) J_{\mu}(\beta)-\beta J_{\mu}(\alpha) J_{\mu+1}(\beta)}{\alpha^{2}-\beta^{2}}
\end{gathered}
$$

Inserting eq. (13) into expression (11) we get for the transverse momentum distribution of a quark in the $(n, \mu)$ quantum state the following expression:

$$
\begin{aligned}
& P_{n \mu}\left(p_{\mathrm{T}}\right)=2 . r_{n \mu}^{2} k_{n \mu}^{2} \frac{\left(2 \pi R^{2}\right)^{2}}{\left(x_{n \mu}^{2}-\beta^{2}\right)^{2}} \\
& \quad \times\left\{\left[x_{n \mu} J_{\mu+1}\left(x_{n \mu}\right) J_{\mu}(\beta)-\beta J_{\mu}\left(x_{n \mu}\right) J_{\mu+1}(\beta)\right]^{2}\right. \\
& \left.\quad+\left[x_{n \mu} J_{\mu}\left(x_{n \mu}\right) J_{\mu+1}(\beta)-\beta J_{\mu+1}\left(x_{n \mu}\right) J_{\mu}(\beta)\right]^{2}\right\},
\end{aligned}
$$

with $\beta=p_{\mathrm{T}} R$ as before. The total transverse momentum distribution of created quarks is then given by the sum over all quantum states $(n \mu)$ :

$P\left(p_{\mathrm{T}}\right)=2 \sum_{n=1}^{\infty} \sum_{\mu=0}^{\infty} P_{n \mu}\left(p_{\mathrm{T}}\right)$.

The factor two before the sum accounts for states with $\pm \mu$. In a similar way we obtained the corresponding expressions for the transverse momentum distribu- tion of the antiquarks and established the equality, $\bar{P}_{n \mu}\left(p_{\mathrm{T}}\right)=P_{n \mu}\left(p_{\mathrm{T}}\right)$, as it is expected due to momentum conservation.

\section{Pair creation in a chromoelectric flux tube}

Let us assume that the excited hadron, the decay of which we study, can be considered as a sufficiently long chromoelectric flux tube of radius $R$, such that the effects due to its finite length are negligible. In this case we can apply the results of ref. [8], and that of the last section to describe its decay. We use an abelian approximation inside the flux tube and replace the electric charge by the effective chromoelectric charge $g_{\mathrm{e}}$ according to $g_{\mathrm{c}}^{2}=\frac{4}{3} g^{2}=\frac{16}{3} \pi \alpha_{\mathrm{QCD}}$. The next step is to replace the field strength $\delta$ by the string tension $\kappa$. In order to do this we use Gauss' law

$g_{\mathrm{c}}=\mathscr{E} \pi R^{2}$

and the physical meaning of the string tension as the linear energy density along the tube:

$$
\begin{aligned}
& \kappa(r)=\left(\frac{1}{2} \mathscr{g}^{2}+B\right) \pi r^{2} \\
& =\left(\frac{1}{2} \frac{g_{\mathrm{c}}^{2}}{\left(\pi r^{2}\right)^{2}}+B\right) \pi r^{2},
\end{aligned}
$$

where $B$ denotes the bag constant. As to the latter, we consider two cases:

Case $A$. With the assumption that the string can always adjust its transverse size to its actual length, it equilibrates at a radius $R$ for which $\kappa$ is smallest. Thus we obtain the relations

$g_{\mathrm{c}} \mathscr{E}=\kappa, \quad R=\left(\frac{16 \alpha_{\mathrm{QCD}}}{3 \kappa}\right)^{1 / 2}$.

Case $B$. Assuming that the vacuum pressure can be neglected with respect to the field energy, we obtain from eqs. (17) and (18) the relations

$g_{\mathrm{e}} \mathscr{E}=2 \kappa, \quad R=\left(\frac{8 \alpha_{\mathrm{QCD}}}{3 \kappa}\right)^{1 / 2}$.

Since the excited hadron is a system far from equilibrium, it should represent a situation somewhere midway between cases A and B. For a realistic model $\alpha_{\mathrm{OCD}}$ should be of the order of or perhaps somewhat larger than $\alpha_{\mathrm{QCD}}\left(Q^{2}=1 \mathrm{GeV}^{2}\right) \approx 0.3$. Thus for case $A$ the radius should be $\gtrsim 0.6 \mathrm{fm}$ and for case $B \gtrsim 0.4$ 
$\mathrm{fm}$. It will turn out that the fits to the $p_{\mathrm{T}}$ distribution and multiplicities favour exactly these values, i.e. correspond to acceptable values for $\alpha_{\mathrm{QCD}}$. According to eqs. (17) and (19) or (20), we can calculate $\alpha_{\alpha \cdot \mathrm{D} \text {, }}$ $\delta$, and $g_{\mathrm{e}}$ for a given string tension $\kappa=0.89 \mathrm{GeV} / \mathrm{fm}$ and radius $R$ in both cases.

The corrections to the pair creation rate due to the finite radius are given in table 1 . With increasing field strength $\mathscr{R}(R)$ increases too. We see that in the range $R \leqslant 1 \mathrm{fm}$ (except the case $g_{\mathrm{c}} \delta=2 \kappa$ and $R=1 \mathrm{fm}$ ) the only significant contribution comes from the creation of pairs in the lowest lying excited state $(n=1$, $\mu=0$ ). In this case the transverse momentum distribution is independent of the string tension $\kappa$. Making use of the linear boundary condition on the surface of the cylinder [8] we can write the transverse momentum distribution in the following form:

Table 1

Correction $\mathscr{R}(R)$ to the pair production rate due to the finite radius $R$ of the flux tube.

\begin{tabular}{|c|c|c|c|c|}
\hline \multirow{2}{*}{$\begin{array}{l}R \\
(\mathrm{fm})\end{array}$} & \multirow[t]{2}{*}{$(n \mu)$} & \multirow[t]{2}{*}{$x_{n \mu}$} & \multicolumn{2}{|l|}{$\not R(R)$} \\
\hline & & & $g_{e} \delta=\kappa$ & $g_{e} \delta=2 \kappa$ \\
\hline \multirow[t]{3}{*}{0.4} & $(10)$ & 1.4347 & & 0.060 \\
\hline & (11) & 2.6299 & & $10^{-6}$ \\
\hline & & & & 0.060 \\
\hline \multirow[t]{3}{*}{0.6} & $(10)$ & 1.4347 & 0.089 & 0.345 \\
\hline & (11) & 2.6299 & $10^{-5}$ & 0.001 \\
\hline & & & 0.089 & 0.346 \\
\hline \multirow[t]{4}{*}{0.8} & $(10)$ & 1.4347 & 0.298 & 0.523 \\
\hline & (11) & 2.6299 & 0.001 & 0.031 \\
\hline & $(20)$ & 3.1129 & $10^{-4}$ & 0.007 \\
\hline & & & 0.299 & 0.561 \\
\hline \multirow[t]{5}{*}{1.0} & $(10)$ & 1.4347 & 0.461 & 0.567 \\
\hline & $(11)$ & 2.6299 & 0.014 & 0.080 \\
\hline & $(20)$ & 3.1129 & 0.002 & 0.030 \\
\hline & (12) & 3.7689 & $10^{-4}$ & 0.005 \\
\hline & & & 0.477 & 0.682 \\
\hline
\end{tabular}

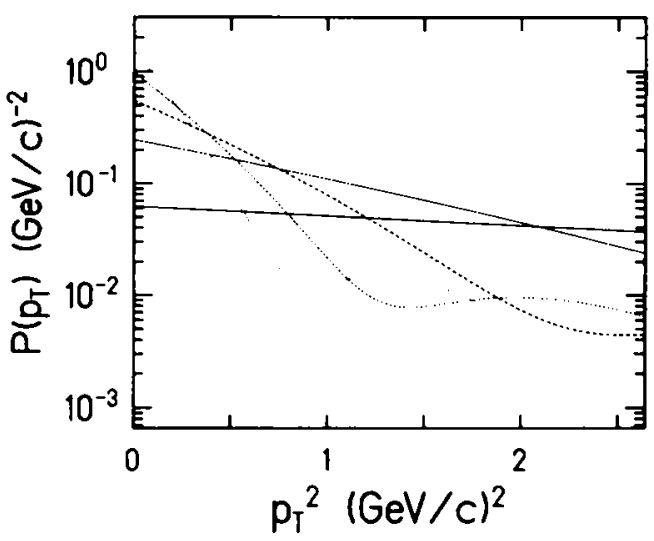

Fig. I. Transverse momentum distribution of (anti)quarks created in the chromoelectric flux tube of radius $R=0.2 \mathrm{fm}$ (thick solid line), $0.4 \mathrm{fm}$ (thin solid line), $0.6 \mathrm{fm}$ (thick dashed line), $0.8 \mathrm{fm}$ (thin dashed linc), and $1.0 \mathrm{fm}$ (dotted line).

$$
\begin{aligned}
& P\left(p_{\mathrm{t}}\right) \approx P_{10}\left(p_{\mathrm{T}}\right) \\
& \quad x\left\{\left[x_{10}^{2}+\left(p_{\mathrm{T}} R\right)^{2}\right]\left[J_{0}^{2}\left(p_{\mathrm{T}} R\right)+J_{1}^{2}\left(p_{\mathrm{T}} R\right)\right]\right. \\
& \left.-4 x_{10} p_{\mathrm{T}} R J_{0}\left(p_{\mathrm{T}} R\right) J_{1}\left(p_{\mathrm{T}} R\right)\right\}\left[x_{10}^{2}-\left(p_{\mathrm{T}} R\right)^{2}\right]^{-2} .
\end{aligned}
$$

The transverse momentum distribution of quarks is shown in fig. 1. One can see that the distribution gets rather forward peaked for increasing radius $R$ in accordance with the uncertainty principle. The transverse momentum distributions in the low- $p_{\mathrm{T}}$ region can be approximated by gaussian distributions, $P\left(p_{\mathrm{r}}\right) \approx \exp \left(-p_{\mathrm{T}}^{2} / 2 p_{\mathrm{T} 0}^{2}\right)$ with the width parameter $p_{\mathrm{T} 0} \approx k_{10}=x_{10} / R$. This does not mean, however, that the width of the transverse momentum distribution of the quarks tends to zero in the limit $R \rightarrow \infty$ since then an increasing number of excited radial states contributes to the pair creation rate.

\section{Fragmentation of hadronic strings}

In the dynamical string model presented in ref. [11] hadrons are considered as string-like extended objects which obey the equations of motion of infinitely thin $(3+1)$-dimensional strings $[12,13]$, but exhibit a finite interaction range $[7,14]$. It is assumed that any infinitesimal string piece decays with the probability $A \mathrm{~d} A$ where $\mathrm{d} A$ is the invariant surface element which has been swept by the string piece and 
$A$ is the decay constant. Then the decay of the string is governed by an exponential decay law [15]. The decay constant is given by $A=n_{\mathrm{f}} \mathbb{H} \pi R^{2}$ where $n_{\mathrm{f}}=3$ is taken as the number of active flavours. For each string an invariant area $A_{0}$ is chosen with the distribution $\exp \left(-\Lambda A_{0}\right)$. Having swept out this area $A_{0}$ on the world sheet, the string starts to decay. A point with uniform distribution is chosen on the string segments whose increment in the invariant area is maximal in the next infinitesimal time step. A virtual qq̃ pair is thought to be created at this point with compensating transverse momenta $\pm \boldsymbol{p}_{\mathrm{r}}$ with directions distributed uniformly in the plane perpendicular to the decaying string piece in its rest frame. The virtual pair departs along the string with the speed of light. After the for- mation time $\tau_{\mathrm{f}}=p_{\mathrm{T}} / \kappa$ has elapsed, i.c. when the energy of the string piece between the virtual pair can cover its transverse energy, the pair appears on-massshell. The string piece is then taken away and the two daughter strings start their motion with transverse momenta at their "new" endpoints. As the distance between the pair cannot be longer than the total length of the flux tube, the magnitudes of the transverse momenta of the created pair are limited by some maximal value $p_{f}^{(\max )}$ depending on the motion of the endpoints of the string.

For the simulation of the hadron production in $\mathrm{e}^{+} \mathrm{e}^{-}$annihilation we assume that the quark and the antiquark created at a single point at the $\mathrm{CM}$ time $t=0$ stretch out a one-dimensional string when de-
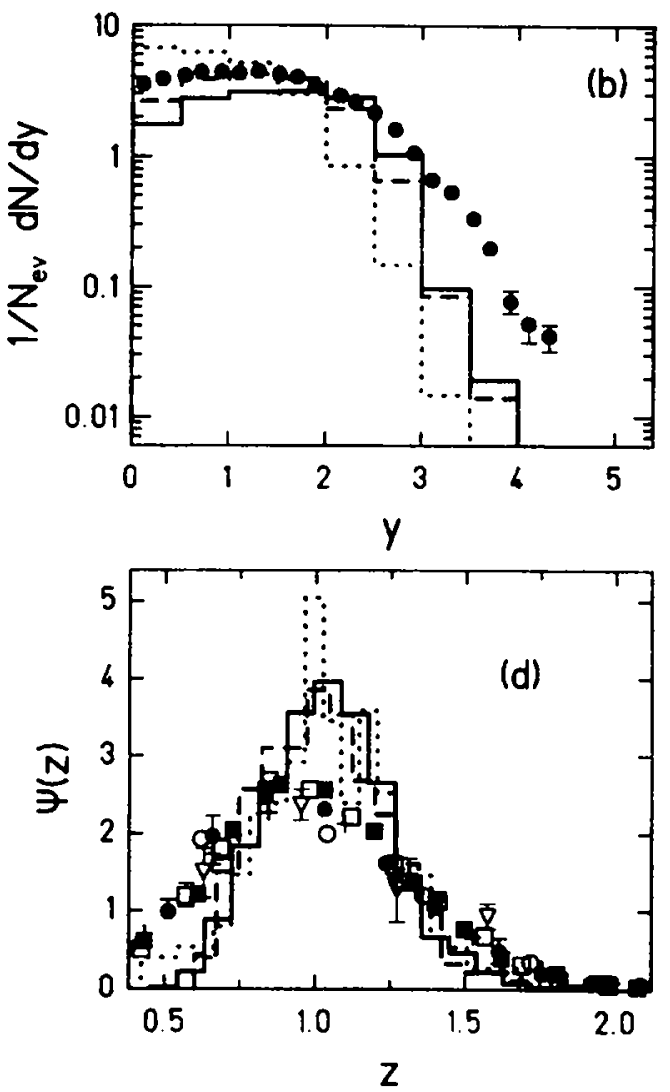

Fig. 2. Simulated (a) $x_{\mathrm{F}}$, (b) rapidity, $y$, and (c) transverse momentum, $p_{\mathrm{T}}$ distributions and (d) KNO scaling functions for hadrons produced in $\mathrm{e}^{+} \mathrm{c}^{-}$annihilation at $20 \mathrm{GeV} \mathrm{CM}$ energy for $g_{\mathrm{e}} \delta=\kappa$ and for the string radius $R=1.0 \mathrm{fm}$ (solid line), $0.8 \mathrm{fm}$ (dashed line), and $0.6 \mathrm{fm}$ (dotted line). Experimental data are taken from ref. [3] and denoted by dots for $22 \mathrm{GeV}$, full and empty quadrants for 34 $\mathrm{GeV}$ and $30.6 \mathrm{GeV}$, respectively, crosses for $14 \mathrm{GeV}$, triangles for $7.4 \mathrm{GeV}$, and circles for $5 \mathrm{GeV} \mathrm{CM}$ energies. 
Table 2

Dependence of the average charged particle multiplicity $\left\langle n_{\mathrm{ch}}\right\rangle$ on the string radius $R$.

\begin{tabular}{lllll}
\hline$g_{\mathrm{e}} \delta=\kappa$ & & & $g_{\mathrm{e}} \delta=2 \kappa$ & \\
\cline { 5 - 6 }$R(\mathrm{fm})$ & $\left\langle n_{\mathrm{ch}}\right\rangle$ & & $R(\mathrm{fm})$ & $\left\langle n_{\mathrm{ch}}\right\rangle$ \\
\hline 0.6 & 11.1 & 0.4 & 12.0 \\
0.8 & 8.9 & & 0.6 & 8.0 \\
\hline
\end{tabular}

parting with the speed of light. In ref. [11] we obtained a good overall agreement with experimental data with exception of the transverse momentum distribution of the fragments, although the pair production rate was not corrected for the finite size effect. The width of the transverse momentum distribution of the fragments was found to be too small in comparison with the data.
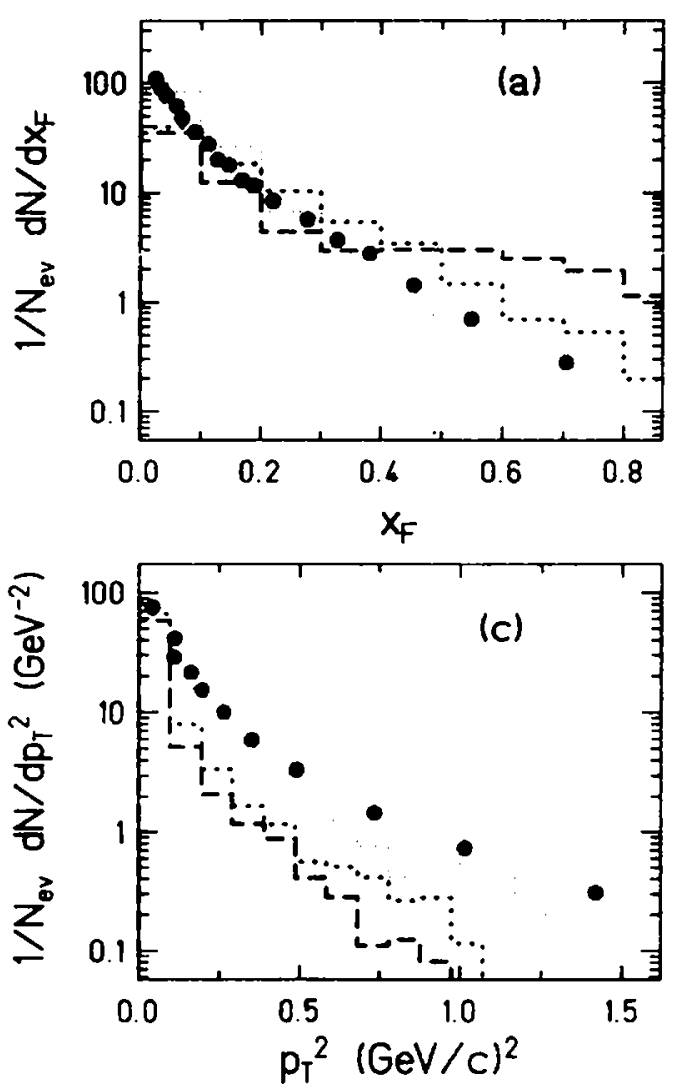

Now we take the finite size effects into account according to the results obtained in ref. [8] and the first part of the present paper. The string radius is considered as a free parameter having the same value for all strings irrespectively of their rest mass. The dependence of the observables on the value of the string radius is studied.

The fragments are characterized by their total fourmomenta, $\left(\epsilon, p_{\mathrm{T}}, p_{z}\right)$, where $p_{\mathrm{T}}$ and $p_{z}$ are the transverse and longitudinal momenta with respect to the jet axis in the $\mathrm{CM}$ frame. The distributions of the fragments in the Feynman variable $x_{\mathrm{F}}=2 p_{z} / \sqrt{s}$, in rapidity $y=\frac{1}{2} \ln \left[\left(\epsilon+p_{z}\right) /\left(\epsilon-p_{z}\right)\right]$, and in the transverse momentum $p_{\mathrm{T}}$ have been determined by a simulation of 500 two-jet events at the invariant energy $\sqrt{s}=20 \mathrm{GeV}$. The time evolution was followed in steps of $\Delta t=0.02 \mathrm{fm} / c$ until the strings became "stable", where the stability threshold $m_{\mathrm{th}} \approx 1 \mathrm{GeV}$ was
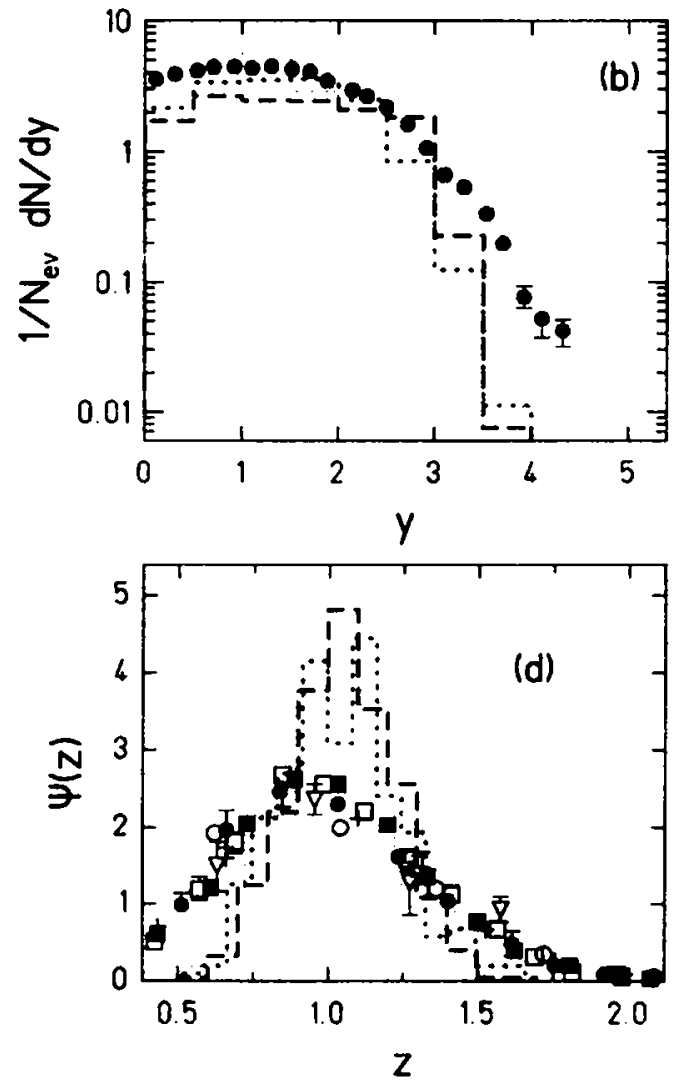

Fig. 3. The same as fig. 2, but for $g_{e} \delta=2 \kappa$ and for $R=0.8 \mathrm{fm}$ (dashed line), $0.6 \mathrm{fm}$ (dotted line), and $0.4 \mathrm{fm}$ (thin dotted line). 
defined by the criterion that the average mass of stable hadrons is identical in the case of a discrete and that of a continuous mass spectrum [11].

The dependence of the simulated $x_{F}$, rapidity, and transverse momentum distributions, and KNO scaling function on the string radius $R$ is shown in figs. $2 a-2 d$ and $3 a-3 d$ for cases $A$ and $B$, respectively. The calculated distributions (with exception of the KNO functions) are multiplied by a factor $\frac{2}{3}$ in order to compare them with the corresponding data on charged particles. We see that the $x_{\mathrm{F}}$ distribution becomes steeper, and the rapidity distribution gets narrower with decreasing radius whereas the transverse momentum distribution of the fragments broadens. With decreasing string radius the mean transverse momentum increases and therefore less energy remains for the longitudinal motion. That explains the change of the shape of the $x_{F}$ and rapidity distributions. The change of the slope of the transverse momentum distribution reflects the uncertainty principle. The simulated KNO scaling function is not sensitive to the string radius and has a smaller width than the data. Radius values $R \in(0.6 \mathrm{fm}, 0.8 \mathrm{fm})$ for case A and $R \in(0.4 \mathrm{fm}, 0.6 \mathrm{fm})$ for case B seem to be appropriate to fit the distributions in figs. 2 and 3 .

In table 2 we present the average charged particle multiplicities $\left\langle n_{\mathrm{ch}}\right\rangle=\frac{2}{3}\langle n\rangle$ calculated from the average fragment multiplicities $\langle n\rangle$. Comparing them with the value $\left\langle n_{\mathrm{ch}}^{\mathrm{exp}}\right\rangle=10.5$ interpolated from experimental data [ 16 ], we find that $R \approx 0.6 \mathrm{fm}$ for case $\mathrm{A}$ and $R \approx 0.4 \mathrm{fm}$ for case $\mathrm{B}$ are the preferred radius values. For these values the average formation time $\tau_{\mathrm{f}}=p_{\mathrm{T} 0} / \kappa \approx x_{10} / \kappa R$ ranges from $0.4 \mathrm{fm} / c$ to $0.8 \mathrm{fm} /$ $c$. Since $t=\tau_{\mathrm{f}}$ is roughly the time in the CM system of the initial string when it breaks up, so in the best case ( $R=0.4 \mathrm{fm}$ in case $\mathrm{B}$ ) its length is twice its diameter at that moment, but never longer. This means that effects due to the finite length of the string can lead to a significant correction to our results.

\section{Concluding remarks}

We have studied the influence of the finite radius of the hadronic string on their fragmentation. The transverse extension has been added to the strings on the basis of flux tube model considerations, without taking into account its effect on the motion of the string. The decay constant and the transverse momentum distribution of the created qā pairs were calculated for a flux tube of finite radius but infinite length. The interaction of the created quarks and antiquarks was neglected. It is found that only the first excited state with radial quantum numbers $(n=1$, $\mu=0$ ) contributes to the pair creation in hadronic strings.

The simulation of two-jet events in $\mathrm{e}^{+} \mathrm{e}^{-}$annihilation at $20 \mathrm{GeV} \mathrm{CM}$ energy has revealed the following features:

(i) Depending on the strength of the chromoelectric field in the excited hadron, a string radius between 0.4 and $0.6 \mathrm{fm}$ allows for a reasonable fit of the $x_{\mathrm{F}}$, rapidity, and $p_{\mathrm{T}}$ distribution of the fragments as well as the mean charged particle multiplicity. The width of the $p_{T}$ distribution is roughly given by the uncertainty principle. This suggests that it is reasonable to describe the longitudinal motion of a hadronic string classically and its transverse oscillations quantum mechanically. Such a picture would be in agreement with the properties of string-like solutions found in long-distance QCD [14].

(ii) For the radii obtained the corresponding formation time is about $1 \mathrm{fm} / c$. Thus the strings generally decay before they get much longer than their diameter. This indicates that the applicability of the string fragmentation model is rather due to the predominance of longitudinal momenta than to the geometrical shape of the excited hadrons. This implies also that our treatment of the pair production is only approximate and corrections like those predicted in ref. [17] can play a role. Consequently our result for the string radius could be somewhat modified by more claborate calculations.

\section{References}

[1] T. Sjöstrand, Intern. J. Mod. Phys. A 3 (1988) 751.

[2] B. Andersson, G. Gustafson, G. Ingelman and T. Sjöstrand, Phys. Rep. 97 (1983) 31.

[3] P. Mättig, Phys. Rep. 177 (1989) 141.

[4] B. Andersson, Z. Phys. C 6 (1980) 235.

[5] F. Sauter, Z. Phys. 69 (1931) 742; J. Schwinger, Phys. Rev. 82 (1951) 664.

[6] A. Casher, H. Neuberger and S. Nussinov, Phys. Rev. D 20 (1979) 179;

N.K. Glendenning and T. Matsui, Phys. Rev. D 28 (1983) 2890. 
[7] J. Wosick and R.W. Haymaker, Phys. Rev. D 36 (1987) 3297 ;

A.B. Migdal, S.B. Khokhlachev and L.N. Shchur, Sov. Phys. JETP 64 (1986) 441.

[8] Th. Schönfeld, A. Schäfer, B. Müller, K. Sailer, J. Reinhardt and W. Greiner, preprint UFTP 242/1990, submitted to Phys. Lett.

[9] I.S. Gradstein and I.M. Ryshik, Tafeln, Vol. 2 (Harri Deutsch, Thun, 1981) p. 479.

[10] E. Jahnke, F. Emde and F. Lösch, Tables of higher functions (Teubner, Stuttgart, 1960) p. 156.

[11] K. Sailer, B. Müller and W. Greiner, Intern. J. Mod. Phys. A 4 (1989) 437; preprint UFTP 230/1989; in: Quark gluon plasma, ed. R.C. Hwa (World Scientific, Singapore, 1990), to appear.
[12] Y. Nambu, Lecture Copenhagen Summer Symp. (1970), unpublished;

O. Hara, Prog. Theor. Phys. 46 (1971) 1549;

T. Gotō, Prog. Theor. Phys. 46 (1971) 1560.

[13] X. Artru, Phys. Rep. 97 (1983) 147.

[14] M. Baker, J.S. Ball and F. Zachariasen, Phys. Rev. D 37 (1988) 1036

[15] X. Artru and G. Mennessier, Nucl. Phys. B 70 (1974) 93.

[16] TASSO Collab., W. Braunschweig et al., Z. Phys. C 45 (1989) 193.

[17] M. Herrmann and J. Knoll, preprint GSI-89-67 (1989). 